\title{
Stage C Prostate Cancer
}

National Cancer Institute

\section{Source}

National Cancer Institute. Stage C Prostate Cancer. NCI Thesaurus. Code C7552.

Tumor is clinically localized to the periprostatic area, but extends through the prostatic capsule; the seminal vesicles may be involved. -- 2004 